\title{
Estilos musicales de los estudiantes universitarios: ¿Oportunidad para el diálogo intercultural?
}

\author{
Musical styles among students: Opportunity for the \\ intercultural dialogue?
}

\author{
Ahtziri Molina Roldán \\ Aldo Colorado Carvajal \\ Instituto de Investigaciones en Educación, Universidad Veracruzana \\ ahtziri@gmail.com \\ alcolorado@uv.mx
}

\section{Resumen}

Este trabajo examina sí el espacio universitario puede ser considerado como promotor de las relaciones interculturales entre los jóvenes estudiantes universitarios con distinto capital cultural y condición social. Este trabajo se pregunta si hay un sentido identitario compartido generado desde la universidad, que pase por la aceptación e incluso apropiación de la diversidad expresada mediante los distintos estilos musicales que los jóvenes escuchan y comparten en este espacio educativo.

El análisis se realiza con base en los resultados de la encuesta usos del tiempo libre y consumo cultural de los estudiantes de la Universidad Veracruzana realizada en 2008, la cual recoge información del $10 \%$ de la población universitaria en todas las regiones del estado.

Palabras clave: Universitarios, capital cultural, estilos musicales, Juventud, interculturalidad

\begin{abstract}
This paper questions if the university as a space of interaction can be considered as promoter of intercultural relations among the young students, who have different cultural capital and social condition. This article inquires if university experience provides a shared identity's sense of acceptance and appropriation of diversity. The previous expressed throughout the musical styles the students listen and share in this educational space.

The analysis is based upon the data collected in the 2008 survey: Leisure and cultural consumption of the Universidad Veracruzana students, which was carried out in 2008 and collects information of the $10 \%$ of the student population from all the areas of the state where the university is present.
\end{abstract}


Key words: Students, cultural capital, musical styles, youth, intercultural.

\section{Introducción}

Los espacios universitarios desde sus inicios han sido identificados como los lugares idóneos para la construcción del conocimiento así como para la transmisión de los postulados vigentes en todas las ramas del saber. Las dinámicas universitarias han hecho del intercambio de ideas, la discusión de paradigmas, el cuestionamiento de sistemas teóricos y la generación de modelos, sus herramientas más importantes para el desarrollo de las tareas. Por lo tanto, para que el conocimiento fluya este debe estar centrado en la comunicación y la conformación de comunidades y estar destinado a ellas. En este artículo consideramos que la Universidad es un espacio basado en el respeto, el derecho a disentir, la aceptación de la diferencia, valores que al menos teóricamente, harían de la universidad un espacio para la interculturalidad por antonomasia.

$\mathrm{Al}$ interior de las universidades, los estudiantes se encuentran entre los principales destinatarios del trabajo generado en las universidades. La formación de profesionistas históricamente ha implicado el desarrollo de dos caminos paralelos que son, la formación profesional en un área de especialización y por el otro la convivencia y acercamiento con materias y actividades que complementan su formación universitaria y humana.

Este artículo considera la capacidad de la universidad como un espacio para la interacción y la negociación de identidades de jóvenes que provienen de distintos contextos socioculturales con sus respectivos estilos musicales Por lo tanto, los ejes reflexivos de este trabajo son: la universidad como posible espacio generador de las relaciones interculturales entre los jóvenes estudiantes universitarios con distinto capital cultural y condición social; quienes se encuentran en un momento decisivo en la construcción de su identidad individual y colectiva.

Los objetivos de este estudio son identificar los estilos musicales más socorridos entre los universitarios como indicador de los grados de interculturalidad generados por la universidad entre los estudiantes en los cinco campus de la Universidad Veracruzana (UV).

Este trabajo asume que el sentido compartido que debe de generar la universidad es la aceptación de la diversidad. Y sí aceptar que la diversidad significa apropiarse de ello, por lo tanto: ¿Es el intercambio de estilos musicales realmente un generador de sentido compartido en el espacio universitario, respetando y valorando las diferencias? 
El análisis se genera a partir de la información compilada en otoño de 2008 (Molina y Casillas Coord ${ }^{1}$ ) mediante una encuesta de usos de tiempo libre y consumo cultural aplicada al 10\% de la población con matrícula 2006. Esta encuesta recabó información sobre estudiantes de todas las regiones de la Universidad Veracruzana. Entre las preguntas se incluyeron los estilos musicales que escuchan con más frecuencia. En el cuestionario se inquirió sobre diecisiete estilos musicales: comerciales, como alternativos y clásicos. Lo anterior con la finalidad de tener una mejor idea del universo musical de los universitarios. El artículo se desarrolla con base en la información estadística y utilizando referentes teóricos de los modelos universitarios, el capital cultural, los estilos musicales juveniles en el marco de la interculturalidad.

\section{Marco analítico}

La Universidad en sus inicios fue concebida como un espacio de generación, preservación y transmisión del conocimiento a nivel superior y profesionalizante. Sin embargo, las formas en que esto ha sido ejecutado han cambiado de acuerdo al tiempo y las sociedades. Brunner, declara que esto tiene que ver con el modelo de universidad que se plantea de acuerdo a los espacios y tiempos dados (2008).

Los principales modelos que han existido son: las universidades medievales fundadas en el poder de la iglesia católica previa al fortalecimiento del Estado nación. Posteriormente el modelo de las universidades europeas que tuvieron distintos parámetros, donde prevalecieron las germánicas o humboldtianas, las cuales fueron moldeadas como corporaciones al servicio de la ciencia y la investigación, lejos de Dios y de alguna otra ideología, permitiendo así la libre búsqueda de la verdad y la transmisión de la cultura nacional. Además de que la búsqueda científica ha de darse en espacios integrales lo cual pone en duda la parcialización del conocimiento y da paso para pensar en la universidad como espacio idóneo para el desarrollo de la cultura universal' ${ }^{2}$.

Este modelo desarrollado a principios del siglo XIX tuvo ecos importantes en la América Latina de comienzos del siglo XX. En 1918 los estudiantes de la Universidad de Córdoba demandan que las instituciones de educación superior fueran autónomas de la Iglesia y de los poderes civiles y que la universidad tuviera una clara función social; además de libertad de expresión y libre acceso a las aulas universitarias (Aboites, 2008). Este movimiento tuvo ecos en otras partes del continente y en México la Universidad Nacional Autónoma de México fue constituida en 1929 por otro gran promotor del conocimiento

\footnotetext{
${ }^{1}$ Este documento se encuentra en revisión final para su publicación.

${ }^{2}$ Cabe la pena mencionar, que mucho tiempo lo universal en América Latina ha sido interpretado como lo proveniente de la cultura occidental anglo-hispano-europea.
} 
universal y la reivindicación de la identidad latinoamericana: José Vasconcelos. En este sentido, el modelo de universidad latinoamericana, cuyo objetivo era reflexionar sobre la realidad local a la par de establecer diálogo con las teorías occidentales -comprendidas como universales- buscaban condiciones para que la interculturalidad fuera posible.

Sin embargo, en el último tercio del siglo XX el impulso neoliberal marcó cambios importantes en la vida universitaria. La puesta en marcha de políticas neoliberales a nivel global cuestionan en las prácticas las formas de ser y hacer la universidad, donde se cuestiona el cuantioso gasto público para el funcionamiento de las instituciones de Educación Superior. Además de que bajo estos nuevos patrones las competencias y certificación homogenizan las formas de educar, y los conocimientos impartidos. (Declaración de Bolonia, 1999).

Las modificaciones en las políticas socio-económicas de la región han impactado directamente la organización y objetivos de la universidad actual, modificando -al menos parcialmente- los valores y prácticas de la comunidad universitaria. En este sentido, las actividades académicas se adaptan a las reglas del mercado y el uso generalizado de las tecnologías informáticas; lo cual impacta la estructura y vida universitaria.

Lo anterior es destacado por el trabajo de Brunner (2007) quien genera marcos de análisis estructurales de las universidades considerando actores y factores internos y externos, no incluye a los estudiantes. Lo anterior concuerda con el hecho de que algunos teóricos sobre la universidad han argumentado que los estudiantes no pueden ser considerados como actores de las instituciones universitarias, pues se encuentran de paso. Lo cual implica que los estudiantes, una de las razones de ser más importantes de las instituciones de educación superior, no son considerados en el análisis estructural de las universidades y esto reduce las posibilidades de que sus opiniones y necesidades sean tomadas en cuenta en la vida universitaria.

El estudio sobre los jóvenes universitarios tanto a nivel mundial como en nuestro país se da como consecuencia del crecimiento masivo de la matrícula universitaria y de su inminente diversificación a partir de la década de los años sesenta. Estos cambios en la población universitaria han dado lugar a una muy importante transformación de la estructura y las representaciones sociales sobre lo que es la universidad y lo que significa ser universitario.

Uno de los rasgos que más han cambiado en las maneras de ser estudiante universitario es el consumo cultural de los jóvenes estudiantes. En efecto, a diferencia del pasado cuando los estudiantes encontraban toda la oferta cultural en la universidad y desde ella se apropiaban de los bienes culturales, ahora esto no es más así. Los estudiantes universitarios llegan a las aulas de las instituciones siendo portadores de muy diversas dotaciones culturales y con un amplio bagaje de disposiciones culturales que han construido en el pasado y cultivado en su vida cotidiana en tanto jóvenes. 
El proceso educativo se está transformando, pues las interacciones ocurren con jóvenes adultos plenos de información, con amplios recursos tecnológicos y cognitivos para procesarla, en espacios de fuerte intercambio multicultural, con uso frecuente de términos provenientes de otros idiomas y de "igualdad" entre estudiantes y profesores.

Por otra parte, en la actualidad se ha definido de muchos modos la noción de joven, sin embargo la que este trabajo elige es la emitida por Rojek (2005) quien plantea que la juventud es una etapa tipificada por responsabilidades y obligaciones sociales ligeras, finanzas limitadas y en general dependencia familiar. Además este autor reconoce a la juventud como:

Un período de experimentación en las áreas de formación de identidad y adquisición de status como individuos absorben modelos a seguir y estímulos de los grupos de amigos y los medios de comunicación, en contraste con valores familiares y comunitarios que hasta ese momento de su vida habían sido los predominantes. Esta es una etapa dominada por el proceso de individualización y desarrollo de identidades sexuales (Rojek, 2005: 126) ${ }^{3}$.

Consideramos esta noción muy completa, pues agrega a las dimensiones sociales de los jóvenes las características psicobiológicas asociadas con la adolescencia, las cuales son sistemáticamente omitidas desde las ciencias sociales. Sin embargo, muchas de las características sociales que 'problematizan' el ser joven y más que ser joven, el control que se ejerce sobre los jóvenes, son de esta naturaleza. Tales serían los casos del inicio a la sexualidad, de la búsqueda de la independencia del grupo familiar o de la construcción identitaria a partir de la influencia de sus pares y la generación de nuevos círculos sociales donde son predominantemente autónomos de sus padres.

Enfatizando los factores sociales, la juventud implica una serie de procesos de experimentación en muchas actividades de la vida humana y formas de socialización que eventualmente derivaran en los cánones elegidos o posibles para funcionar en la adultez. Entre ellas destacan: las pautas de preparación para el empleo, el establecimiento de relaciones afectivas y la creación de redes exteriores a las instituciones familiares y escolares que les permitan a los sujetos socializar y desarrollar su capital cultural a lo largo de sus vidas.

En la actualidad, uno de los elementos más importantes para la construcción identitaria de los jóvenes es su paso por las subculturas juveniles ${ }^{4}$, las cuales representan un vehículo de transformación social, con códigos, formas y expresiones muy específicas, tales como los estilos musicales, la moda, las expresiones corporales, el argot, entre las principales.

\footnotetext{
${ }^{3}$ Traducción propia

${ }^{4}$ Cabe la pena aclarar, que se le han nombrado de modos distintos en diferentes estudios y hay quien rechaza totalmente esta acepción.
} 
Dicho énfasis ha llevado a esta vertiente del conocimiento a hacer del estilo la fuente principal para estos estudios; los cuales incluyen entre sus intereses, el lenguaje, los estudios etnográficos concernientes al espacio, las manifestaciones de cultura popular y los significados como ha sido el caso de Hebdige (1979).

Sin embargo, a diferencia de los años iniciales donde las identificaciones de los jóvenes con el estilo elegido tendían a ser absolutas, ahora las elecciones de estilo suelen ser menos ancladas en algún estilo específico debido a la fragmentación y proliferación de géneros musicales. Lo anterior como resultado de la diversificación y popularización de los estilos juveniles y por el gran cúmulo de información al que están expuestos los jóvenes hoy en día y que manifiestan en sus prácticas cotidianas de interacción especialmente en los clubes nocturnos. A esto Polhemus (1996) le ha denominado stylesurfing; a lo cual agrega Muggleton: "Ellos no tienen que preocuparse por las contradicciones entre las identidades subculturales elegidas, porque no hay reglas, no hay autenticidad, ni compromiso ideológico, meramente un juego estilístico en el cual participar" (2000: 47). Por lo tanto, estos jóvenes tienen la posibilidad y capacidad de moverse entre distintas elecciones y estar más abiertos a las distintas tendencias presentadas.

Una variable importante en la elección e identificación de los estilos musicales y el desarrollo de la identidad individual de los sujetos es el cúmulo de capital cultural con el que llegan a la universidad. La noción de capital cultural fue acuñada por Bourdieu (1987) para conformar una unidad de medida que explicara el diferente desempeño escolar de los estudiantes de acuerdo con sus disposiciones culturales y no sólo sus diferencias económicas. No se trata de negar la existencia de diferencias económicas entre los individuos y grupos sociales sino de observar éstas en el terreno específico de la cultura y la educación (Casillas, Chain y Jácome, 2007).

Esta noción se refiere a las distintas dotaciones culturales de las cuales los agentes son poseedores y que utilizan para sus interacciones escolares como recursos para avanzar su desempeño. Se trata de conocimientos, maneras de ser y de comportarse, de disposiciones incorporadas que diferencian a los individuos; también se trata de bienes simbólicos que en forma de certificados o diplomas distinguen a sus poseedores, pues no sólo son los grados, áreas de conocimiento o tipo de cursos consignados, sino el valor de las instituciones el que objetivan los diplomas; finalmente, también se trata de recursos y de condiciones materiales de existencia asociadas con los estudios y los conocimientos, recursos que no sólo se expresan en tanto objetos sino que implican también su dominio (libros, equipos electrónicos y de cómputo, etcétera). La hipótesis de base es que las prácticas de consumo cultural estarán asociadas a las distintas dotaciones del capital cultural y por tanto a las condiciones y estilos de vida de los grupos sociales.

\footnotetext{
${ }^{5}$ Traducción propia.
} 
Para este trabajo, recuperamos el trabajo de Casillas, Chain y Jácome (2007) donde operacionalizan la noción de capital cultural a través de una tipología de estudiantes universitarios. Con los mismos recursos hemos actualizado la información de los distintos grupos y preparado para la muestra una tipificación específica para este estudio.

Tomando en cuenta su capital cultural, analizamos también la trayectoria escolar de los estudiantes. La cual se construyen con base en la aprobación y reprobación, la promoción y el rezago, el promedio obtenido y el grado de avance esperado según los programas de estudio.

Con base en el modelo de universidad contemporánea, los estilos juveniles, el capital cultural y las trayectorias escolares, analizamos sí las preferencias musicales de la población en cuestión están desarrollando un diálogo intercultural en el espacio universitario.

\section{Antecedentes}

En otoño de 2009, un 10\% de la población de la generación 2006 de la Universidad Veracruzana contestó un cuestionario sobre los usos que hacen de su tiempo. Se prestó especial atención a las actividades que forman parte de sus prácticas cotidianas en su vida como universitarios. En este cuestionario se incluyeron siete temas de consumo cultural y uso del tiempo libre: práctica y asistencia a actividades artísticas, usos de las tecnologías, actividades deportivas, de participación social, formativas, recreativas y festivas. Una de las secciones de tecnologías estuvo dedicada al tipo de música que escuchan y la frecuencia con lo que lo hacen. Es mediante estas preferencias musicales que deseamos saber sí han modificado sus prácticas y elecciones musicales a partir de las experiencias y actividades que desarrollan en el espacio universitario.

A nivel nacional, Adrian de Garay se ha encargado de estudiar y sistematizar los perfiles de los jóvenes estudiantes a través de diversas caracterizaciones socio-económicas y culturales. Lo ha realizado mediante distintos estudios que cubren a las Instituciones de Educación Superior afiliados a la ANUIES Los actores desconocidos (2001), la integración de los jóvenes en el sistema universitario (2004) y los perfiles académicos y socioculturales desarrollados por los estudiantes de las Universidades Tecnológicas (2006). El autor caracteriza a los estudiantes de acuerdo a las actividades que realizan, los espacios donde se llevan a cabo y las frecuencias con que las desarrollan.

En el caso particular de la UV, se han desarrollado varios estudios sobre las trayectorias escolares del estudiantado (Chain, 1995) y la elección de carreras (Chain, 2001). Además de un análisis sobre el capital cultural de los estudiantes de nuevo ingreso (Casillas, Chain y Jácome, 2007). Esta última investigación retoma los fundamentos en los trabajos 
sociológicos de Bourdieu y Passeron (1977). Cabe además señalar que estos trabajos han sido desarrollados con base en la información provista por la base de datos sistematizada por el sistema COINPI ${ }^{6}$. Fue sobre esta base que analizamos los aspectos socioeconómicos de esta investigación.

\section{Contexto}

La UV está presente en cinco regiones del Estado de Veracruz. Dada la posición estratégica de este Estado y en particular de sus puertos como punto de entrada del mundo occidental, en este territorio se han desarrollado un amplio número de culturas y formas de vida que abrevan tanto de las influencias externas como de los espacios donde se han establecido.

La peculiar geografía del Estado de Veracruz que cubre una amplia parte del litoral del Golfo de México hacen que se presenten diversas orografías, afluentes y culturas en los 71 $820 \mathrm{~km} 2$ que tiene la totalidad de su territorio (aregional.com, 2009), las cuales se han dividido en seis zonas geográfico-culturales que incluyen costa, montaña, mesetas y selvas, con sus respectivas expresiones culturales. Guardadas las distancias, la situación geográfica del estado y su división política es comparable con la chilena en una escala de 1/8 de la extensión total del país sudamericano.

Para el conteo de población 2005, el estado tenía una población de 7,110,214 habitantes, los cuales habitan en 212 municipios. El 60.7\% de la población vive en zonas urbanas, entre las cuales destacan las de: Tuxpan- Poza Rica, Xalapa-Coatepec, VeracruzBoca del Río, Córdoba-Orizaba y Coatzacoalcos-Minatitlán.

En lo que a educación se refiere, Veracruz es una entidad con enormes rezagos, hay un número muy grande de analfabetas, el rezago escolar es una condición muy extendida entre la población y la proporción de estudiantes universitarios es una de las más bajas a nivel nacional. La distribución de la población que se disemina en miles de pequeñas comunidades rurales hace muy difícil brindar servicios educativos, en el marco de un estado muy extendido y con una difícil orografía. Sin embargo, también la situación educativa es propia del grado de desarrollo del Estado: Veracruz es una de las entidades del país con más pobres; miles de niños cursan la primaria en los Cursos Comunitarios del CONAFE, complementado con un numeroso sistema de telesecundarias y de telebachilleratos. De ese circuito de educación precaria proviene un número muy grande de nuestros estudiantes universitarios, los cuales son portadores de las disposiciones

\footnotetext{
${ }^{6}$ Sistema de Consulta Individualizada del Perfil de Ingreso (COINPI) contiene la información de todos los estudiantes que ingresaron a la Universidad Veracruzana y permite consultar la información detallada de cada estudiante.
} 
culturales que han podido construir a lo largo de su experiencia escolar en esos contextos educativos.

\section{La Universidad Veracruzana}

En cuanto a educación superior se refiere, desde 1944, la Universidad Veracruzana es la máxima casa de estudios del Estado. La UV fue creada simultáneamente en distintas localidades del largo Estado de Veracruz, sin embargo, la rectoría general está ubicada en Xalapa y esto implica que una parte importante del aparato universitario se encuentra en esta ciudad capital.

Tanto por sus alcances académicos, como por la cobertura que tiene la UV es catalogada por la ANUIES como una institución de educación superior orientada a la transmisión, generación y aplicación del conocimiento, que ofrece programas en el nivel de técnico superior, licenciatura, maestría y doctorado. La institución cuenta con seis áreas académicas: Artes, Ciencias Biológico-Agropecuarias, Ciencias de la Salud, Económicoadministrativas, Humanidades y Técnica en las cuales se imparten 69 carreras en las modalidades, escolarizada, abierta y a distancia.

Esta institución, que es la de mayor tamaño e importancia en el estado de Veracruz tiene alrededor de 55 mil estudiantes inscritos en licenciatura ${ }^{7}$ quienes se forman en los cinco campus situados a lo largo del estado y los cuatro de la Universidad Intercultural. Con esto, la Universidad tiene presencia en los principales centros urbanos del estado y en zonas con importante presencia indígena y de agricultores.

A partir de 1998 instituyó el Modelo Educativo Integral y Flexible (MEIF) el cual tiene como objetivos: "Propiciar en los estudiantes de las diversas carreras que ofrece la Universidad Veracruzana una formación integral: intelectual, humana, social y profesional". Lo anterior con las finalidades de desarrollar habilidades, destrezas, actitudes y valores, para lograr: aprobación, desarrollo de valores humanos, sociales y culturales, un pensamiento lógico, crítico y creativo, el establecimiento de relaciones interpersonales y de grupo. Así como un óptimo desempeño en la auto-formación permanente (Nuevo Modelo Educativo para la Universidad Veracruzana, 1999).

A diferencia de la mayoría de las instituciones mexicanas y anticipándose históricamente a los tiempos, la Universidad Veracruzana desde su fundación en 1944, se ha preocupado por desarrollar, fomentar y sustentar una oferta cultural amplia y variada que represente las diferentes manifestaciones culturales del pueblo veracruzano y también las de carácter universal (Casillas, 2008). Además de contar con un potente proyecto de

\footnotetext{
${ }^{7}$ Numeralia UV 2010. http://www.uv.mx/numeralia/ Universidad Veracruzana.
} 
difusión cultural, es una institución que tiene un área de conocimiento propia para las artes donde se agrupan facultades y programas muy variados ${ }^{8}$.

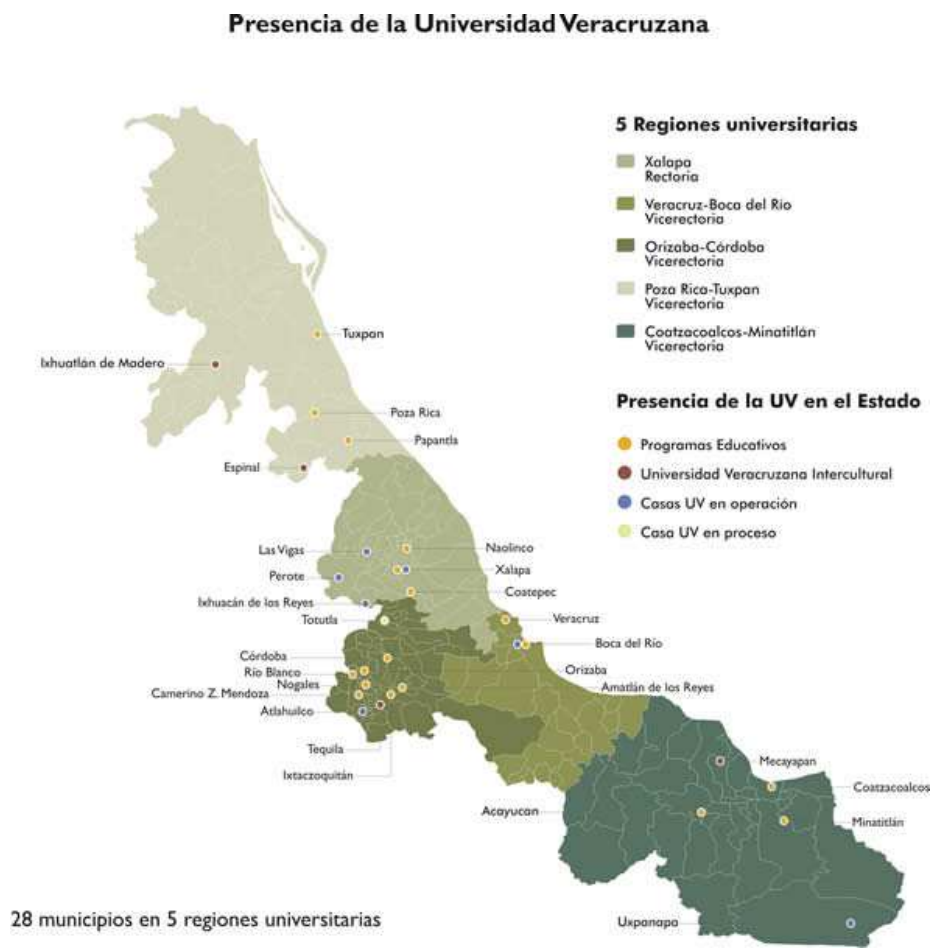

Fig 1. Sedes de la Universidad Veracruzana

Asociado al signo de los tiempos, con la emergencia de una dinámica empresa cultural, con el desarrollo de las nuevas tecnologías y con la propia condición juvenil de los estudiantes contemporáneos, en años recientes observamos en nuestra encuesta la baja participación de los estudiantes en muchas de las actividades artístico-culturales promovidas por esta casa de estudios. No obstante que, en general, todos los estudiantes declaran escuchar música con frecuencias altas, como a continuación se presenta.

\section{Análisis estadístico}

\section{Música}

\footnotetext{
${ }^{8}$ Para mayor información ver: http://www.uv.mx/artes/
} 
La encuesta sobre consumo cultural y uso del tiempo se aplicó a una muestra de 1,087 estudiantes que representaban el $10 \%$ de aquellos que ingresaron a la Universidad en el año 2006. Mediante un cuestionario electrónico se recabó la información de los estudiantes y, se construyó la base de datos resultante.

En términos generales, el 60\% de los encuestados eran mujeres y 40\% hombres. Sólo una quinta parte de los estudiantes declaró que practicaban o estaban aprendiendo a tocar un instrumento musical; apenas la mitad dijo que practicaba un deporte y dos terceras partes que asistían a eventos artísticos. En general, los estudiantes realizan pocas actividades, aunque sobresalen en actividades recreativas y festivas como pasear, asistir a centros nocturnos o bares o a las plazas comerciales. Sólo alrededor del 20\% realizan alguna actividad extraescolar, como sería tomar algún curso o pertenecer a algún grupo social.

El uso de las tecnologías ha llevado a nuevas formas de socialización y de interacción social de los jóvenes; los estudiantes, como jóvenes, participan de estos procesos tecnológicos y sociales que inciden en el uso que le dan a su tiempo en general. 2 de cada 5 estudiantes pasan de 3 a 6 horas diarias frente a la computadora e internet.

Como ya mencionamos, escuchar música es una de las actividades de esparcimiento más importantes que realizan los jóvenes universitarios, es de fácil acceso y su costo es mínimo. A pesar de esto, escuchar música ya no es de las actividades más frecuentes que realicen los estudiantes, ya que actualmente pasan más tiempo frente a la computadora donde, de igual manera, muchos de ellos tienen acceso a la música (60\% dijo que utiliza la computadora para bajar o escuchar música).

Los jóvenes universitarios escuchan la radio de 1 a 2 horas al día, y principalmente escuchan programas musicales ( $80 \%$ frecuente y muy frecuentemente). La música acompaña la realización de las tareas domésticas (46\%), siguiendo aquellos que la escuchan sólo por el gusto de escucharla $(44 \%)$ y por último, quienes que la escuchan mientras usan la computadora (39\%) y los que la escuchan mientras se transportan (38\%). Mientras que las jóvenes escuchan la música para acompañar otras actividades; los hombres, por su parte, escuchan la música por el gusto de escucharla. Aquellos

La tecnología ha permitido llevar la música a todas partes y escucharla mientras se realiza cualquier actividad, lo que ha provocado que los estudiantes ya no tengan que ir a espacios específicos para escuchar música. Esto puede observarse en que sólo el $60 \%$ de los estudiantes declaró que van a conciertos o festivales musicales, y de éstos, 3/4 partes de ellos lo hace ocasionalmente, es decir, con frecuencia de una vez al mes o menos. De igual forma, los estudiantes no asisten a otro tipo de eventos musicales como los raves o tocadas, sólo $5 \%$ asiste a los primeros y el $20 \%$ a los segundos. Por su parte, salir a bailar o al antro, se realiza con mayor frecuencia, $40 \%$ de los estudiantes mencionó realizar esta actividad. 
La mayoría escucha música comercial o masiva, $75 \%$ de los estudiantes escuchan música pop, $64 \%$ rock en español, $62 \%$ rock en inglés, $47 \%$ boleros y baladas y $45 \%$ reggaetón. Contra aquellos que escuchan música clásica (30\%) o jazz-blues (18\%).Estos últimos géneros son identificados con expresiones musicales cercanas a la academia y a una preparación musical formal, como las que produce y difunde la Universidad Veracruzana.

\section{Capital Cultural}

Consideramos que el consumo cultural, en este caso a través del consumo musical, se define por ciertos recursos culturales y disposiciones de los individuos que les permiten acercarse a uno u otro estilo de música. Por lo tanto, observar estas dotaciones de capital cultural que poseen los estudiantes, es necesario para ir acercándonos a entender el consumo musical de los estudiantes.

Como ya se mencionó, el capital cultural es aquel válido dentro del espacio cultural y educativo que se observa en los bienes o disposiciones incorporadas, que poseen los estudiantes y que les sirven para desenvolverse en la escuela.

De esta forma, sólo el $40 \%$ de los universitarios provienen de espacios socio-familiares que cuentan con un alto capital cultural (herederos), un 20\% aproximadamente son estudiantes con alto capital escolar pero deficiente capital familiar (héroes y pobres exitosos) y, por último, otro $40 \%$ de jóvenes con orígenes sociales precarios y de marginación y con capital escolar deficiente 9 .

Tabla 1. Capital cultural de los estudiantes universitarios

\begin{tabular}{lll} 
Capital cultural & Frecuencia & Porcentaje \\
\hline Herederos & 328 & 37.6 \\
Héroes & 45 & 5.2 \\
\hline Pobres exitosos & 160 & 18.3 \\
\hline Riesgo & 211 & 24.2 \\
\hline Alto riesgo & 129 & 14.8 \\
\hline Total & 873 & 100.0 \\
\hline
\end{tabular}

${ }^{9}$ Para más detalles sobre la metodología usada para la construcción del capital cultural ver Casillas, Chain y Jácome (2007). 
Herederos. Este grupo lo forman estudiantes con altas dotaciones de capital escolar y familiar, son estudiantes que han desarrollado trayectorias escolares exitosas, incluso en el bachillerato y que tuvieron una alta nota en el examen de ingreso a la universidad. Sus padres tienen estudios superiores, desempeñan profesiones y con un alto nivel socioeconómico marcado por altos ingresos e infraestructura en casa.

Héroes. Este grupo estudiantes se distingue por que son excelentes estudiantes, tienen altos promedios de calificaciones del nivel precedente y sacaron una alta nota en el examen de ingreso a la universidad. Sin embargo provienen de espacios sociales altamente marginados, con padres sin escolaridad, generalmente agricultores u obreros. Cuentan con ingresos familiares muy bajos y viven en casas desprovistas de servicios y bienes básicos.

Pobres exitosos. Son estudiantes con desempeños y trayectorias escolares buenas, con buenos promedios en el nivel medio. Se distinguen por que provienen de espacios sociales pobres, son hijos de padres con oficio de empleado. Viven en zonas urbanas, aunque pobres, cuentan con los servicios básicos en sus casas y con una buena cantidad de bienes básicos.

Riesgo. Son estudiantes que tienen bajas trayectorias escolares, con promedios bajos en el nivel medio, con reprobación y con bajas calificaciones en su examen de ingreso. Provienen de espacios socio-familiares pobres, con padres con educación básica o media, con bajos ingresos familiares y con padres con oficio de medio prestigio social, como burócratas o empleados.

Alto Riesgo. Este grupo de estudiantes tiene trayectorias escolares deficientes, con bajos promedios, reprobación y bajas calificaciones en el examen de ingreso. Provienen de espacios familiares marginados y muy pobres, con padres bajo nivel de escolaridad, con bajos ingresos familiares y con oficios de los padres de bajo prestigio social.

De esta forma, esperaríamos que a las diferentes dotaciones de capital cultural que poseen los estudiantes se asociaran a diferentes consumos musicales mediante estilos diversos de música.

\section{Uso del tiempo}

Otra de las características que consideramos de los estudiantes es el uso que hacen de su tiempo respecto a varias actividades consideradas como las principales entre esta población. La intención es observar cómo distribuyen su tiempo y a partir de ello, caracterizarlos según el tiempo dedicado a: estudiar, trabajar, tiempo libre, dormir, transportarse, computadora, internet y televisión. 
De esta forma, tenemos un grupo de estudiantes que se caracterizan principalmente por dedicar buena parte de su tiempo al tiempo libre y dormir (estudiante hedonista 5\%). En segundo lugar, tenemos un grupo de estudiantes que comparten sus estudios con el trabajo (estudiante trabajador 7\%), en estas actividades se distribuye principalmente su tiempo. El tercer grupo, aquellos denominados estudiantes inter-telenautas (12\%), se caracteriza porque es el que comparte su tiempo de estudios con estar frente a la computadora, navegar en internet y ver la televisión más de 4 horas diarias en promedio. El siguiente grupo, una cuarta parte de los estudiantes, responden al tipo ideal de estudiante que la universidad se ha construido (estudiantes de tiempo completo 24\%). Por último, el grupo más numeroso son estudiantes que dedican el tiempo indispensable a la escuela, no trabajan y aunque marcan tener poco tiempo libre, tampoco están frente a la computadora o televisión (estudiante desenganchado 50\%).

Tabla 2. Tipos de estudiantes según el tiempo que dedican a actividades

\begin{tabular}{lll} 
Tiempo de actividades & Frecuencia & Porcentaje \\
\hline Trabajador & 78 & 7.2 \\
\hline Desenganchado & 546 & 50.2 \\
\hline Tiempo completo & 261 & 24.0 \\
\hline Hedonista & 63 & 5.8 \\
\hline Inter-telenauta & 139 & 12.8 \\
\hline Total & 1087 & 100.0 \\
\hline
\end{tabular}

Si bien existe diversidad entre los estudiantes universitarios a partir de cómo distribuyen su tiempo, es importante recalcar que la mitad de los estudiantes parece no comprometido con una actividad en específico, o poco interesados por alguna, por eso los identificamos como desenganchados.

\section{Estilos Musicales}

Pensando en los diferentes estilos de música que escuchan los estudiantes y que en gran parte derivan tanto de sus diferentes capitales culturales como de su pertenencia social a una geografía particular, se decidió agrupar los diferentes estilos musicales que escuchan los estudiantes para identificar algunas tendencias.

Encontramos aquí 5 tipos de estudiantes por los estilos de música que escuchan; el primero, el más grande ( $27 \%$ de los estudiantes) que podemos caracterizar como orientado al estilo comercial y masivo. El segundo grupo, que se puede caracterizar como 
rockero (22\%). El tercer grupo, orientado a la música folklórica y regional (19\%). El cuarto, con una orientación a música rítmica y bailable (18\%). Por último, quienes escuchan música de "alta cultura" (12\%).

Tabla 3. Tipos de estudiantes por estilos musicales que escuchan

\begin{tabular}{|c|c|c|}
\hline Gustos musicales & Frecuencia & Porcentaje \\
\hline Rock en español e inglés y pop & 244 & 22.4 \\
\hline Pop & 294 & 27.0 \\
\hline $\begin{array}{l}\text { Pop, reggae, ska, hip-hop, reggaetón, rock en español e } \\
\text { inglés, electrónica }\end{array}$ & 196 & 18.0 \\
\hline $\begin{array}{l}\text { Pop, rock español, ranchera, grupera, tropical, rock en } \\
\text { inglés, balada, reggaetón }\end{array}$ & 213 & 19.6 \\
\hline Rock en inglés, jazz, clásica & 140 & 12.9 \\
\hline Total & 1087 & 100.0 \\
\hline
\end{tabular}

Cómo podemos observar tenemos 5 grupos, de los cuales, en 4 la presencia de la música pop es importante, así como del rock en español e inglés. La exposición a una cultura masiva y homogeneizante genera estilos musicales compartidos en los estudiantes universitarios, sólo algunos de ellos, los menos, consumen el tipo de música que regularmente oferta la universidad (jazz-clásica) o algún otro ritmo alternativo a los presentes en el mercado.

\section{Tipos de Estudiantes}

Pensando en estos 3 ejes de análisis: capital cultural, uso de tiempo y estilos musicales, construimos una última tipología que resumiera la combinación de las características de los estudiantes.

El primer grupo, estudiantes con alto capital cultural que dedican poco tiempo a las diferentes actividades cotidianas y que escuchan música pop, tradicional, folklórica y masiva-comercial. El segundo grupo, estudiantes que tienen bajo capital escolar, con antecedentes escolares de reprobación y promedios de calificaciones bajos, de ambientes socioeconómicos medios-bajos, que dedican poco tiempo a las actividades cotidianas de los estudiantes y que escuchan música Pop.

El siguiente grupo, es de las mismas características de capital cultural y de uso del tiempo que el precedente pero su consumo musical se enfoca en la música pop, tradicional, folklórica y masiva-comercial. El grupo número 4, son estudiantes con alto capital cultural individual y familiar: con buenos promedios de calificaciones, sin 
reprobación, con padres altamente escolarizados y con altos niveles socioeconómicos, que dedican poco tiempo a las diferentes actividades cotidianas y con estilos orientados al rock y rock-pop. El último grupo, son estudiantes de alto capital cultural, que dedican una parte considerable de su tiempo a navegar en el internet y ver televisión y que orientan sus preferencias musicales al pop, tradicional, folklórica, masiva y comercial.

Tabla 4. Tipo de estudiantes universitarios por capital cultural, uso del tiempo y estilos musicales

\begin{tabular}{|c|c|c|c|c|}
\hline & $\begin{array}{l}\text { Capital } \\
\text { cultural }\end{array}$ & $\begin{array}{l}\text { Uso del } \\
\text { tiempo }\end{array}$ & Estilos musicales & $\begin{array}{l}\text { Porcenta } \\
\text { je }\end{array}$ \\
\hline $\begin{array}{l}\text { Grupo } \\
1\end{array}$ & $\begin{array}{l}\text { Heredero } \\
\mathrm{S}\end{array}$ & $\begin{array}{l}\text { Desenganchad } \\
\text { os }\end{array}$ & $\begin{array}{l}\text { Pop, rock español, ranchera, } \\
\text { grupera, tropical, rock en inglés, } \\
\text { balada, reggaetón }\end{array}$ & 15 \\
\hline $\begin{array}{l}\text { Grupo } \\
2\end{array}$ & Riesgo & $\begin{array}{l}\text { Desenganchad } \\
\text { os }\end{array}$ & Pop & 36 \\
\hline $\begin{array}{l}\text { Grupo } \\
3\end{array}$ & Riesgo & $\begin{array}{l}\text { Desenganchad } \\
\text { os }\end{array}$ & $\begin{array}{l}\text { Pop, rock español, ranchera, } \\
\text { grupera, tropical, rock en inglés, } \\
\text { balada, reggaetón }\end{array}$ & 15 \\
\hline $\begin{array}{l}\text { Grupo } \\
4\end{array}$ & $\begin{array}{l}\text { Heredero } \\
\mathrm{S}\end{array}$ & $\begin{array}{l}\text { Desenganchad } \\
\text { os }\end{array}$ & Rock en español e inglés y pop & 21 \\
\hline $\begin{array}{l}\text { Grupo } \\
5\end{array}$ & $\begin{array}{l}\text { Heredero } \\
\mathrm{S}\end{array}$ & Inter-telenautas & $\begin{array}{l}\text { Pop, rock español, ranchera, } \\
\text { grupera, tropical, rock en inglés, } \\
\text { balada, reggaetón }\end{array}$ & 12 \\
\hline
\end{tabular}

De esta forma, encontramos grupos amplios de estudiantes que varían muy poco entre sí; es una diversidad homogénea, uniformante y uniformadora la que caracteriza a los estudiantes universitarios. Los jóvenes actualmente están más expuestos a los mass media que generaciones anteriores, lo cual ha derivado en procesos de socialización más o menos homogéneos, esto es evidente al observar las características de los estudiantes por la música que escuchan.

\section{Análisis}

Cuando iniciamos esta investigación consideramos que, debido a la diversidad cultural, étnica y geográfica del estado y la presencia de la Universidad en cinco regiones, los estilos musicales elegidos por los jóvenes serían muy variados. Sin embargo, los datos no reflejan esta diversidad. La variedad que se presenta entre ellos es baja y por lo tanto esto reduce las posibilidades de compartir estilos musicales y experiencias diferentes. Es más, este análisis apunta al hecho de que no tienen siquiera el interés o herramientas necesarias para 
incorporar a su vida estilos musicales variados o que si lo hacen, es a nivel superficial, como propone el stylesurfing.

Para explicar este fenómeno nosotros consideramos las siguientes razones: La penetración de los medios masivos de comunicación a todos los sectores de la población es bastante efectiva, por lo tanto se presenta como primera opción para el desarrollo de los gustos musicales más socorridos entre los estudiantes; a pesar de provenir de contextos sociales distintos. Sin embargo, las condiciones económicas, las condiciones de acceso a los medios masivos y los estilos de vida asociados a la globalización parecen tener más peso en la conformación identitaria individual que los contextos y capitales socioculturales en los que estos jóvenes se formaron previos a su ingreso a la Universidad. Además hay que tomar en cuenta que el $60 \%$ de los estudiantes de nuestra universidad cuentan con capitales culturales bajos, lo cual los sitúa en desventaja social para aumentar su capital cultural con prácticas tales como: tener mayor acceso a la tecnología, realizar viajes, acceder a eventos diversos, entre otros.

Tampoco parecen estar muy presentes las motivaciones personales que por su condición de jóvenes les condujera a buscar alternativas en la conformación de sus identidades. La falta de diversidad en este espacio y momento de la vida puede deberse a que la universidad aun representa -para el sector que ingresa a ella- una entrada al espacio laboral y por ende adulto. Por otra parte, hay que recordar que se les entrevistó a la mitad de la carrera por lo cual puede ser que estos jóvenes estén en la búsqueda de un perfil más adulto, las opciones musicales distintas se dejen de lado, con la finalidad de ingresar al mercado laboral y otras responsabilidades que implica la vida adulta.

Además consideramos que la oferta universitaria existente no busca establecer un diálogo con las preferencias musicales y los estilos de vida de preferencia de los estudiantes, lo cual se demuestra a partir de los bajos índices de preferencia manifestados por los estudiantes en los estilos musicales que promueve la Universidad.

Al parecer los cambios en la organización universitaria impactaron directamente la posibilidad de que esta institución se viva como un espacio universal, conformador de profesionistas integrales. En la UV dos condiciones modificaron internamente las posibilidades de que la institución genere actividades culturales relevantes para los jóvenes que promuevan el diálogo intercultural: la desarticulación y consecuente atomización y pauperización de las instancias artísticas de la propia institución y la introducción del MEIF.

En nombre de las competencias y la eficiencia terminal -valores y preceptos más ligados al mercado que a la formación humana- este modelo rompió con las lógicas de generación, lo cual dio al traste con las oportunidades de convivir e intercambiar experiencias y formas de vida entre los estudiantes. La distribución individualizada que ahora hacen los estudiantes con sus tiempos, así como la poca actividad artística 
disponible desde sus centros de estudio no permiten una mayor interacción ni la posibilidad de buscar alternativas más allá de los horarios y formas establecidas. La falta de convivencia, así como la lógica de alcanzar los valores del mercado global homogenizantes por naturaleza- dificultan las posibilidades de que con este modelo se celebre y promueva la diversidad cultural. Por lo tanto, en este sentido la Universidad no parece estar brindando las oportunidades para diversificar el capital cultural de los educandos, mucho menos de aumentarlo.

Más aún, cabe señalar que la relación universidad-estudiante es de carácter hegemónico y vertical y no ha entablado diálogo con los estudiantes. El tipo de estilos musicales descritos por los estudiantes y los pocos que aseguran escuchar jazz y música clásica nos señalan que la propuesta de la Universidad no está llegando al estudiantado y que peor aún no tiene idea de cómo dialogar con ellos, desde los estilos musicales que les gustan. Esta discrepancia entre realidad y oferta habla de que la Universidad desconoce los gustos de sus estudiantes o si lo hace, ha hecho caso omiso y no hay esfuerzo alguno para entablar el diálogo.

En este sentido cabe recapitular que la figura de los estudiantes está ausente dentro de los grandes esquemas de análisis de la Universidad como institución educativa con un rol específico. Por lo tanto no existen como actores sociales de la universidad, sino como objetos recipiendarios de la producción universitarios. Como se muestra en los textos de Brunner (2007) y Arizpe (2006).

Aunado a lo anterior, es posible pensar que en realidad no es el modelo de humboldtiano, abierto, dispuesto a la diversidad el que permea en esta casa de estudio, sino más bien, uno que enarbolando las banderas de la cultura universal y la cultura local han hecho de esta institución un recinto que legitime desde lo académico expresiones artísticas como lo clásico, jazz-blues y el folklore. Sin embargo, esta legitimación no pasa por el contacto con los estudiantes, quienes desconocen o no gustan de estas opciones.

Por lo tanto, este estudio concluye que en las condiciones actuales, la UV no puede ser considerada como un lugar propicio para el diálogo intercultural; al menos a partir del parámetro de los estilos musicales que utilizamos como referentes. La oferta pop, rock y grupera -las cuales se asocian ampliamente con el mercado- tienen ganada la partida y a pesar de que los jóvenes conviven y se identifican con estos géneros los espacios para ofertas alternativas se ven muy mermados en instituciones donde tradicionalmente habían encontrado una buena acogida.

Lo anterior representa un retroceso importante en la producción y promoción de formas alternativas de expresión, por ende si se mantiene el ideal humboldtiano de formación integral, las universidades requieren conocer e incorporar las visiones y gustos de los jóvenes con la finalidad de que su oferta sea mejor recibida y a través de esto se puedan construir canales de descubrimiento y comunicación con mundos diversos. 
Hay una necesidad de reinventar estos espacios con la utilización de códigos que les sean familiares para adentrarse en mundos más amplios. Lo anterior puede ser mediante acciones desacralizantes del sector como promover concursos de canto, de baile pop, que eventualmente les permita abrir otras puertas y dialogar con otras formas de ser y ver la vida.

\section{Referencias bibliográficas}

\section{LIBROS}

Arizpe, L. (2006). Culturas en Movimiento: Interactividad cultural y procesos globales. México: H. Cámara de Diputados, VXI Legislatura; UNAM; CRIMI; Miguel Ángel Porrua.

Bourdieu, P. y Passeron, J. C. (1977). La Reproducción. Elementos para una teoría de la enseñanza. Barcelona: Laia.

Brunner, J.J. (2007). Universidad y sociedad en América Latina. México: Universidad Veracruzana, Instituto de Investigaciones en Educación.

Casillas, M. y Domínguez Suárez, J.L. (2007). Aproximación a una historia de la Universidad Veracruzana. México: En prensa.

Chaín Revuelta, R. (1995). Estudiantes Universitarios. Trayectorias Escolares. México: Universidad Veracruzana, Universidad Autónoma de Aguascalientes.

Chain Revuelta, R. (2001). Demanda, estudiantes y elección. México: UV-SIGOLFO.

De Garay Sánchez, A. (2001). Los actores desconocidos. Una aproximación al conocimiento de los estudiantes. Colección Biblioteca de la Educación Superior. México: ANUIES.

De Garay Sánchez, A. (2004). Integración de los jóvenes en el sistema universitario. Prácticas sociales, académicas y de consumo cultural. Colección Educación Superior en América Latina. México: Ediciones Pomares Barcelona.

De Garay Sánchez, A. (2006). Las trayectorias educativas en las Universidades Tecnológicas. Un acercamiento al modelo educativo desde las prácticas escolares de los jóvenes universitarios. México: Universidades Tecnológicas, Universidad Tecnológica de la Sierra Madre.

Hedbige, D. (1979). Subculture: The meaning of Style. London: Methuen \& Co.

Muggleton, D. (2000). Inside Subcultur. The postmodern Meaning of Style. Berg: Oxford.

Polhemus, T. (1996). Style surfying: What to wear in the $3^{\text {rd }}$ Millenium. Thames \& Hudson. London.

Rojek, C. (2005). Leisure Theory. Principles and Practice, Londres: Palgrave, Macmillan.

\section{REVISTAS:}

Aboites, Hugo. (2008, Enero). El fututo de la Universidad en América Latina. Revista Acción Educativa. Universidad Autónoma de Sinaloa, No. 8, pp. 6-16. 
Casillas, M. Chaín, R. y Jácome, Nancy. (2007, Abril-Junio). “Origen social de los estudiantes y trayectorias estudiantiles en la Universidad Veracruzana”. Revista de la Educación Superior. No. 142, pp. 7-29.

\section{ARTÍCULOS EN LÍNEA:}

Declaración de Bolonia (1999). En: http://www.bolognaberlin2003.de/pdf/bologna_declaration.pdfConsultado en mayo 2010

INSTITUO NACIONAL DE ESTADÍSTICA, GEOGRAFÍA E INFORMÁTICA (2005) II Conteo de Población y Vivienda 2005, México. En: http://www.inegi.org.mx/est/contenidos/proyectos/ccpv/cpv2005/default.aspx Consultado en Mayo 2010

Monografía del Estado de Veracruz. (2009, Mayo). En: www.aregional.com Consultado en Diciembre 2009

Portal de la Universidad Veracruzana. Área Académica de Artes. (2009). En: http://www.uv.mx/artes/. Consultado en Mayo de 2010

Universidad Veracruzana (1999). Nuevo modelo educativo para la Universidad Veracruzana. Lineamientos para el nivel de licenciatura. Propuesta. Xalapa: Universidad Veracruzana. Disponible http://www.uv.mx/transparencia/infpublica/ofertaacad/documents/nuevo_modelo_educat ivo_lin.pdf 